\title{
Mapping Pigments in a Painting with Low Frequency Electron Paramagnetic Resonance Spectroscopy
}

\author{
Shane McCarthy, Haley Wiskoski and Joseph P. Hornak *D \\ RIT Magnetic Resonance Laboratory, Chester F. Carlson Center for Imaging Science, \\ Rochester Institute of Technology, Rochester, NY 14623-5604, USA; sm6414@rit.edu (S.M.); \\ haleywiskoski@gmail.com (H.W.) \\ * Correspondence: jphsch@rit.edu
}

check for updates

Citation: McCarthy, S.; Wiskoski, H.; Hornak, J.P. Mapping Pigments in a Painting with Low Frequency Electron Paramagnetic Resonance Spectroscopy. Heritage 2021, 4, 1182-1192. https://doi.org/10.3390/ heritage 4030065

Academic Editor: Costanza Miliani

Received: 11 June 2021

Accepted: 7 July 2021

Published: 10 July 2021

Publisher's Note: MDPI stays neutral with regard to jurisdictional claims in published maps and institutional affiliations.

Copyright: (c) 2021 by the authors. Licensee MDPI, Basel, Switzerland. This article is an open access article distributed under the terms and conditions of the Creative Commons Attribution (CC BY) license (https:// creativecommons.org/licenses/by/ $4.0 /)$.
Abstract: An electron paramagnetic resonance (EPR) mobile universal surface explorer (MOUSE) was recently introduced for noninvasively studying paramagnetic pigments in paintings. This study determined that the EPR MOUSE could map the spatial locations of four pigments in a simple impasto painting. Results from three spectral identification algorithms were examined to assess their ability to identify the pigments using an unsupervised approach. Resulting pigment maps are displayed as colorized images of the spatial distribution of the pigments. All three algorithms produced reasonable representations of the painting. The algorithms achieved excellent true positive, true negative, false positive, and false negative rates of $\geq 0.95, \geq 0.98, \leq 0.02$, and $\leq 0.05$, respectively, for the identification of the pigments. We conclude that the EPR MOUSE is suitable for accurately mapping the location of paramagnetic pigments in a painting.

Keywords: low-frequency electron paramagnetic resonance spectroscopy; LFEPR; EPR; mapping pigments; EPR mobile universal surface explorer; EPR MOUSE

\section{Introduction}

Electron paramagnetic resonance (EPR) spectroscopy is a spectroscopy like visible absorption, Raman, and x-ray fluorescence spectroscopies. EPR spectroscopy is a form of magnetic resonance similar in some respects to nuclear magnetic resonance spectroscopy and magnetic resonance imaging. EPR is based on the absorption of electromagnetic radiation at frequency $v$ by unpaired electrons in free radicals and transition metal complexes when experiencing an applied magnetic field B. Materials with unpaired electrons are paramagnetic, hence the origin of the spectroscopy name. EPR spectroscopy is typically performed by keeping $v$ fixed and varying $B$ to find the frequency and magnetic field combination that satisfies the resonance condition

$$
\mathrm{h} v=\mathrm{g} \beta \mathrm{B}
$$

where $\mathrm{h}$ is Planck's constant, $\beta$ the Bohr magneton, and $g$ the Lande g-factor of the specific unpaired electron in the sample. Many, but not all, materials with cultural heritage significance are paramagnetic and possess an EPR signal. These include complexes of transition metals such as copper, manganese, and iron and free radicals such as ultramarine and carbon-based blacks. Conventional high-frequency EPR spectroscopy, operating at $v \approx 9 \mathrm{GHz}$, has been used in archeology and art conservation to study rock [1] and wall paintings [2], ceramics [3-6], pigments [7-11], marble and limestone objects [12-14], paper $[15,16]$, and varnishes used on paintings $[17,18]$. Unfortunately, conventional EPR is invasive, requiring a small amount of the artwork or artifact to be removed for the analysis. EPR is nondestructive in that the sample can be reanalyzed by another analytical technique, but the sampling process has minimally altered the original artwork or artifact. 
Low-frequency EPR (LFEPR) [19] and the novel EPR mobile universal surface explorer (MOUSE) [20] overcome the invasive limitation of conventional EPR, allowing the analysis of the surface of any size artwork or artifact to be performed noninvasively and nondestructively. LFEPR has been demonstrated on linseed oil paints with paramagnetic pigments similar to those used in renaissance paintings. Previous studies have focused on identifying pigments in single-component [21] and double-component [22] paint samples, in addition to layered samples [22], on canvas. Another study addressed imaging the spatial distribution of single paramagnetic and ferromagnetic components [23]. This study addresses mapping the spatial distribution of multiple pigments in a painting on canvas.

The pigment mapping followed an image processing approach used in medical imaging that consists of sampling, identification, and presentation steps [24]. The first step in the process is sampling or collecting the LFEPR spectra point-by-point at regular grid locations on the painting. The resultant data cube is a spatial-spatial-spectral representation of the painting. Sampling is followed by identification of the pigment at each grid location. Identification reduces the three-dimensional spatial-spatial-spectral data into a two-dimensional spatial-spatial map for each pigment in the painting. This was performed by spectral identification algorithms described later. In the final step, presentation, the pigment maps are converted into a single image with one picture element (pixel) for each grid location. This approach is considered unsupervised mapping, as spectra are provided, and a segmented image is produced without subjective human input on the segmentation process [25].

A simple four-color painting with blue vitriol (BV), Han blue (HB), rhodochrosite (RC), and terracotta red (TR) pigments was chosen to demonstrate the utilization of the EPR MOUSE to map the location of pigments in a painting. These pigments were selected because they were paramagnetic and possessed broad, challenging-to-interpret, LFEPR absorption signals. See Figure 1 for an image of the painting.

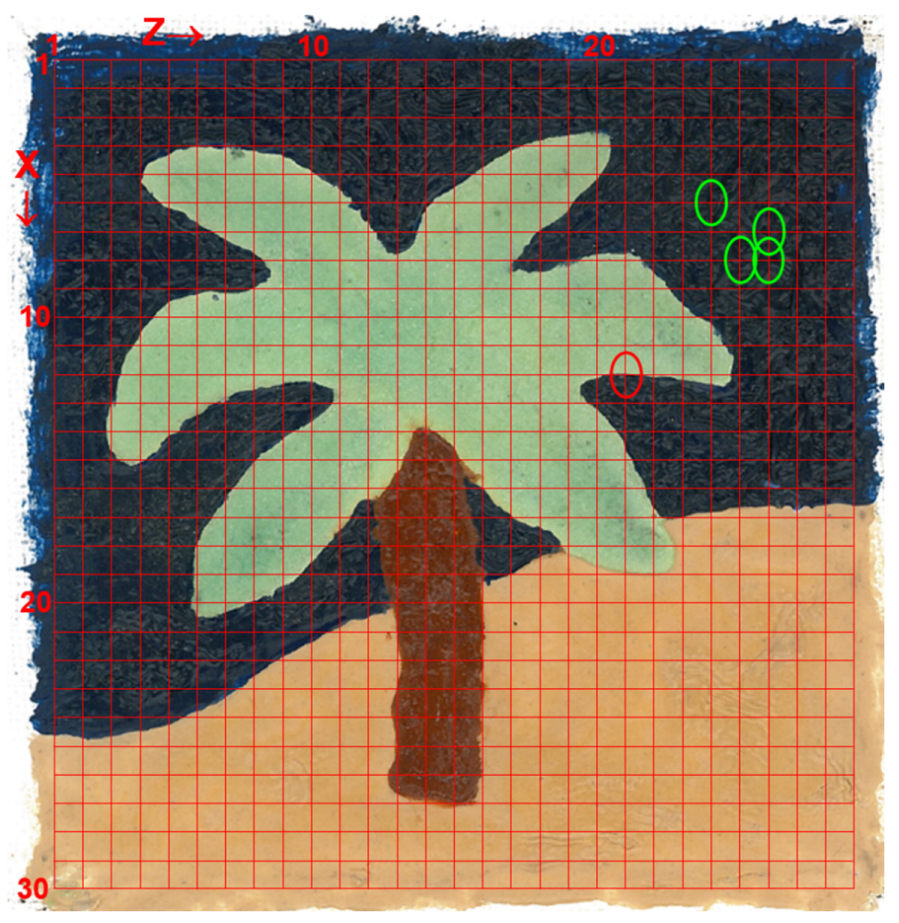

Figure 1. An image of the $10 \times 10 \mathrm{~cm}$ painting Palm Tree on a Beach at Twilight composed of four pigments, (rhodochrosite, terracotta red, blue vitriol, and Han blue) used to demonstrate the ability of the EPR MOUSE to identify and map the location of the pigments. Superimposed on the image is the $Z X$ sampling grid and a few ellipses depicting the size of a sampling location. Ellipses are drawn to indicate the size of the $3.5 \times 5 \mathrm{~mm}$ sampling region. See text for details. 


\section{Background}

The EPR MOUSE is a unilateral LFEPR spectrometer subsystem consisting of the radiofrequency (RF) coil, B field magnet, and modulation magnetic field coils [20] (see Figure 2). The signal is detected from a volume adjacent to the RF coil. The surface of the sampling region is a $3.5 \times 5 \mathrm{~mm}$ ellipse, and the sampling depth is greatest at the center of the ellipse.

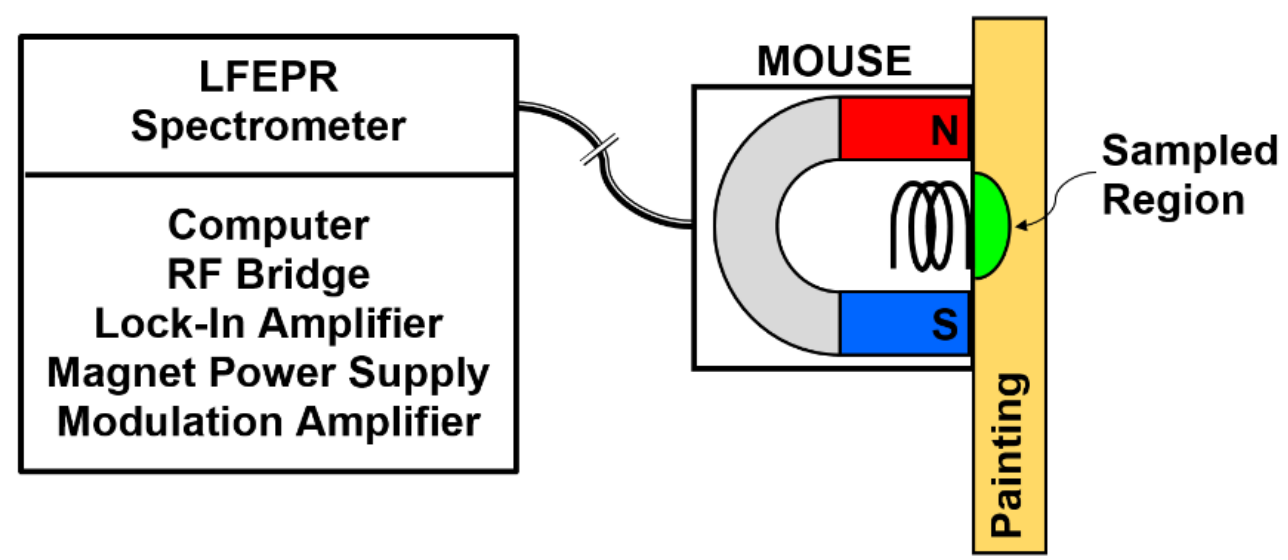

Figure 2. A conceptual drawing of the components of the MOUSE, their connectivity to the LFEPR spectrometer, and the sampled region in the painting.

Unlike other surface analysis techniques where the signal detection depth is dependent on the penetration depth of the incident radiation, the RF, B, and modulation fields all influence detection depth with the EPR MOUSE. The penetration depth for the RF used in LFEPR can be one meter in some materials, but the detection depth is 0 to $1 \mathrm{~mm}$ because of the geometry of the RF, B, and modulation fields.

The MOUSE is connected to the LFEPR spectrometer for the source of $v$, signal detection, and the electromagnetic current. The connection to the spectrometer is via two small variable length cables. The variable length of these cables allows the MOUSE to be positioned meters from the LFEPR spectrometer, and thus analyze any size of painting.

LFEPR spectra represent the absorption of energy at $v$ as a function of $B$. The amount of $B$ examined is referred to as the magnetic field sweep width. Spectra are presented in a first derivative mode owing to the signal acquisition method and hardware [26]. The high-frequency EPR spectral absorptions of paramagnetic pigments tend to be broad. In an LFEPR spectrum, these absorption widths can be comparable to the sweep width of $B$ and can be cut off in the spectrum at the minimum and maximum B sweep values. Figure 3 presents examples of three first derivative LFEPR absorptions. The spectral baseline becomes more difficult to ascertain as the absorption width increases. The lack of a clear spectral baseline creates a challenge for algorithms used to identify the pigment represented by an absorption. Qualitative and quantitative determinations become difficult.

For this reason, three different identification algorithms were examined for the analysis of the painting to determine if one performed best on spectra with baseline uncertainty. They are referred to as the matrix, least squares, and interior-point algorithms. These algorithms were chosen because they represent a range of computational speed and complexity in the order listed. 


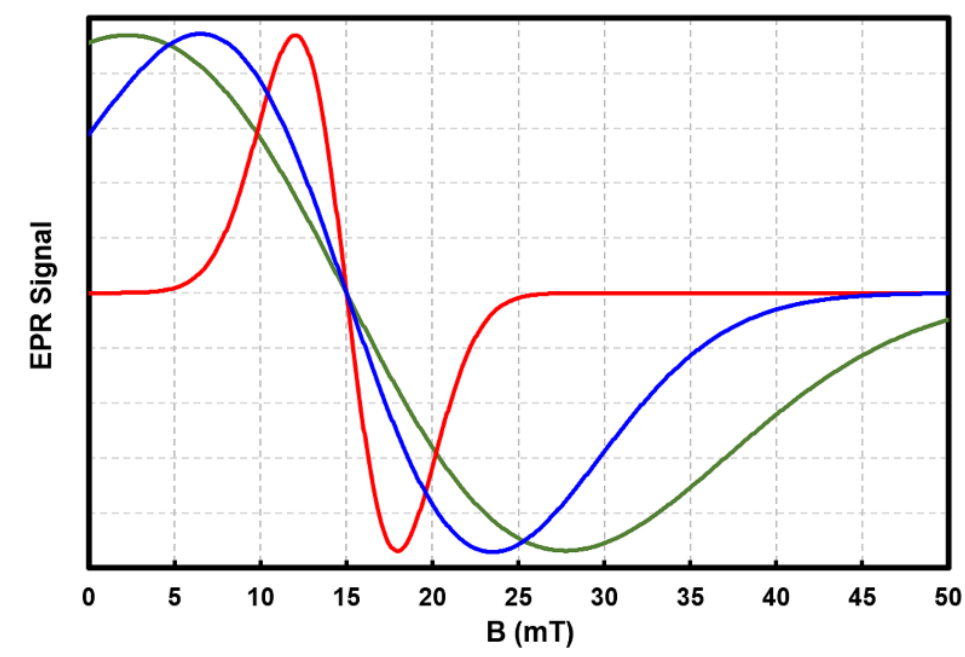

Figure 3. Simulated first derivative EPR absorptions with different widths. Uncertainty in the baseline location increases as the linewidth increases.

Each algorithm assumes the painting is composed of $i$ pigments, each with a unique EPR spectrum $\left(R_{i}\right)$. The total EPR spectrum $(S)$ from a location in the painting is the weighted sum of the spectra from the pigments, where $A_{i}$ is the weighting factor.

$$
S=\sum_{i} R_{i} A_{i}
$$

The matrix algorithm is based on the premise that $i$ equations are needed to solve for $i A_{i}$ values. Defining $S_{m}$ and $R_{i, m}$, respectively, as the total and pigment EPR signals at magnetic field value $m$, Equation (2) becomes

$$
S_{m}=\sum_{i} R_{i, m} A_{i}
$$

Writing Equation (3) in matrix form

$$
\left[S_{m}\right]=\left[R_{i, m}\right]\left[A_{i}\right]
$$

and solving for $\left[A_{i}\right]$

$$
\left[A_{i}\right]=\left[R_{i, m}\right]^{-1}\left[S_{m}\right]
$$

where $\left[R_{i, m}\right]^{-1}$ is the inverse of matrix $\left[R_{i, m}\right]$. Our system of four pigments $(i=\mathrm{RC}, \mathrm{HB}$, $\mathrm{TR}$, and BV) requires spectral values at four field values ( $m=1,2,3$, and 4$)$. The expanded version of Equation (5) becomes

$$
\left[\begin{array}{c}
A_{R C} \\
A_{H B} \\
A_{T R} \\
A_{B V}
\end{array}\right]=\left[\begin{array}{llll}
R_{R C, 1} & R_{H B, 1} & R_{T R, 1} & R_{B V, 1} \\
R_{R C, 2} & R_{H B, 2} & R_{T R, 2} & R_{B V, 2} \\
R_{R C, 3} & R_{H B, 3} & R_{T R, 3} & R_{B V, 3} \\
R_{R C, 4} & R_{H B, 4} & R_{T R, 4} & R_{B V, 4}
\end{array}\right]^{-1}\left[\begin{array}{l}
S_{1} \\
S_{2} \\
S_{3} \\
S_{4}
\end{array}\right] .
$$

Our matrix algorithm is the simplest of the three and placed no constraints on $A_{i}$ solutions.

The least squares algorithm determines which $R_{i}$ is closest to $S$. It assumes one pigment per image location and disregards $A_{i}$ by requiring all spectra be initially normalized. The output is the $i$ value that yields the minimum of the expression

$$
\sum_{m}\left(R_{i, m}-S_{m}\right)^{2}
$$


The interior-point [27] algorithm finds the best $A_{i}$ values that minimize the difference between $A_{i}, R_{i, m}$, and $S_{m}$ at all points $m$ in the spectrum. This algorithm is the most computationally intense of the three.

Each algorithm is applied to spectra from locations on a grid in the painting to create $m$ pigment maps of the painting. Both the matrix and interior-point algorithms are capable of producing pigment maps proportional to the amount of pigment, $A_{i}$. However, this is not possible to achieve with an impasto painting. Although the sampling region is a $3.5 \times 5 \mathrm{~mm}$ ellipse, the pole pieces of the unilateral magnet yoke and surrounding MOUSE housing create a $9 \mathrm{~cm}$ diameter circular flat surface, which is placed against the painting. Depressions in the topography of the painting possess a lower signal than high points in contact with the RF coil. The ability to detect a signal from the MOUSE diminishes to approximately $20 \%$ by $500 \mu \mathrm{m}$ from the RF coil $[20,28]$. For these reasons, all pigment maps were binary, where the largest $A_{i}$ value for a grid location is assigned a value of one in its pigment map.

\section{Materials and Methods}

As a test of the proposed method, a simple four-color $10 \times 10 \mathrm{~cm}$ impasto painting on canvas was prepared using linseed oil paints (see Figure 1). The impasto painting was chosen because it presented a challenging test of the algorithms and for convenience. The paint was hand mulled using boiled linseed oil (Houston Art) and the pigments blue vitriol $\left(\mathrm{CuSO}_{4} \cdot 5 \mathrm{H}_{2} \mathrm{O}\right.$, J.T. Baker), Han blue $\left(\mathrm{BaOCuO}\left(\mathrm{SiO}_{2}\right)_{4}, \mathrm{Kremer}\right.$ Pigments), terracotta red (from fired red clay containing $\mathrm{Fe}^{3+}$ ), and rhodochrosite $\left(\mathrm{MnCO}_{3}, \mathrm{Kremer}\right.$ Pigments). as previously described [22]. The painting, Palm Tree on a Beach at Twilight, is of a palm tree growing in sand against a dark blue sky. The palms of the tree were painted with blue vitriol, which attains a green color when mixed with the oil and dried, the tree trunk with terracotta red, the sky with Han blue, and the sand with rhodochrosite. A contact profilometer was used to characterize the pigment thickness variations in the painting.

Composite images were created from the results to enable comparison to the original painting. A composite image is a color image created from a pigment's component map and its red, green, and blue (RGB) values. Since only one pigment is assigned to a pixel, there is no overlap of colors, and the composite image contains only pixels of four colors.

The collection of the LFEPR signals in the painting was performed using the EPR MOUSE. The painting was attached to an acrylic bed of a two-axis positioning system with $0.1 \mathrm{~mm}$ resolution. The positioning system moves the painting over the MOUSE to the various sampling positions on the $Z X$ grid and lowered it onto the MOUSE. The grid is relative to the MOUSE magnetic resonance coordinate system where $B$ is along $Z$ and the RF polarized along $Y$ [20]. The center of the elliptical sampling region was positioned on a square grid, with a $3.33 \mathrm{~mm}$ spacing between horizontal and vertical grid lines, to produce a $29 \times 30$ point sampling space on the painting. A finer grid is unnecessary, as resolution is limited by the $3.5 \times 5 \mathrm{~mm}$ elliptical sampling region. Locations on the painting are referred to by their $(z, x)$ location. Some overlap between adjacent sampled grid locations existed. Figure 1 presents the grid and a few of the elliptical sampling regions superimposed onto the painting. This image shows the smaller $Z$ and larger $X$ overlap of adjacent ellipses, as exemplified by the ellipses at $(24,8),(25,8)$ and $(25,7),(25,8)$, respectively. When the ellipse spans more than one pigment, as in the ellipse on grid $(21,12)$, fractional sampling occurs, and the EPR spectrum is a weighted sum of spectra from the pigments in the ellipse.

LFEPR spectra were acquired at each grid point using the acquisition parameters presented in Table 1. Acquisition of data from the 870 points on the $29 \times 30$ grid took approximately 14.5 hours. Parameters were chosen to record in a short amount of time a discernable spectrum upon which a determination of the pigment could be made. Spectra were recorded of the four pure pigments and used as reference spectra for the various identification algorithms. Pigments with more similar spectral g-factors and absorption line shapes and widths should be recorded slower with more spectral points to capture their subtle differences, which would take more time. 
Table 1. LFEPR acquisition parameters.

\begin{tabular}{cc}
\hline Parameter & Value \\
\hline Frequency $(v)$ & $388.5 \mathrm{MHz}$ \\
Magnet sweep width & $51 \mathrm{mT}$ \\
Scan time & $13 \mathrm{~s}$ \\
Number of spectral points & 100 \\
Modulation frequency & $10 \mathrm{kHz}$ \\
Modulation amplitude & $2 \mathrm{mT}$ \\
Time constant & $0.1 \mathrm{~s}$ \\
Number of averages & 1 \\
\hline
\end{tabular}

This grid and the ellipses were used to create a digital truth image of the dominant pigment at each location. Dominance was determined through visual assessment of the surface area fraction of a pigment in the ellipse. Those locations covering a surface area fraction greater than 0.6 were assigned the pigment with the larger fraction. Those locations with fractions between 0.6 and 0.4 were assigned the thicker pigment. The truth image is an approximate representation of the visual amount of pigment at each location. Although the EPR signal is not equal to the visual amount owing to variations in the thickness of a paint and its dependence on the concentration in a volume, the top surface of which is only visible, it is the closest available indication of the presence of a pigment. A composite truth image was created using the dominant pigment map and the RGB fractional values of $\operatorname{BV}(0.59,0.73,0.53), \mathrm{HB}(0.00,0.00,0.10), \operatorname{RC}(0.89,0.73,0.50)$, and $\operatorname{TR}(0.47,0.23,0.14)$. This image is presented in Figure 4.

The sampling procedure created a $29 \times 30 \times 100$-point, spatial-spatial-spectral set of data. Each of the spatial points was analyzed by three algorithms for identification of the pigment at the point. The matrix, least squares, and interior-point algorithms were implemented in MATLAB (Mathworks). All algorithms produced four pigment maps where each grid location was single valued. The final step, presentation, was equivalent for each algorithm. The four pigment maps were converted into a single composite image by first colorizing each pigment map with its RGB values. The four layers representing each pigment map were then combined to show the locations of all four pigments in a single RGB image. Creating the composite image was also realized in MATLAB code.

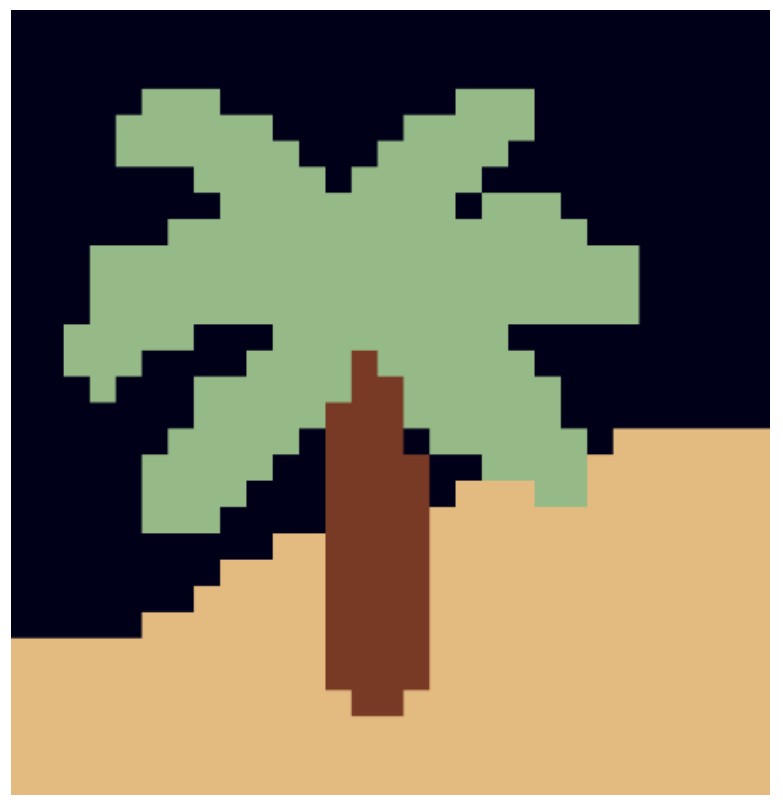

Figure 4. Truth image representing the dominant pigment at each sampling location. 
Four metrics assessed the ability of each algorithm to segment the pigments: true positives (TP), true negatives (TN), false positives (FP), and false negatives (FN) [29]. TP are the number of correctly identified pigment grid points. FP are the number of grid points incorrectly identified as the pigment. TN are the number grid points correctly identified as not being a pigment. FN are the number of pigment grid points that were not identified as the pigment. Because the number of pigment grid points $(\mathrm{P})$ and non-pigment grid points $(\mathrm{N})$ differ for each pigment, ratios were used to facilitate the comparison of an algorithm's ability to identify a pigment. The ratios are the true positive rate (TP/P), false negative rate $(\mathrm{FN} / \mathrm{P})$, true negative rate $(\mathrm{TN} / \mathrm{N})$, and false positive rate $(\mathrm{FP} / \mathrm{N})$.

\section{Results and Discussion}

Figure 5 presents a plot of pigment thickness along the vertical $Z=9$ grid line $3 \mathrm{~cm}$ from the left edge of the painting. The three broad flatter regions with a thickness of $\sim 0.8$ $\mathrm{mm}$ correspond to the blue vitriol in the palm tree foliage. The topography of the impasto painting varied by as much as $600 \mu \mathrm{m}$, making it impossible to perform quantitative determinations with the algorithms. For this reason, all pigment maps were binary.

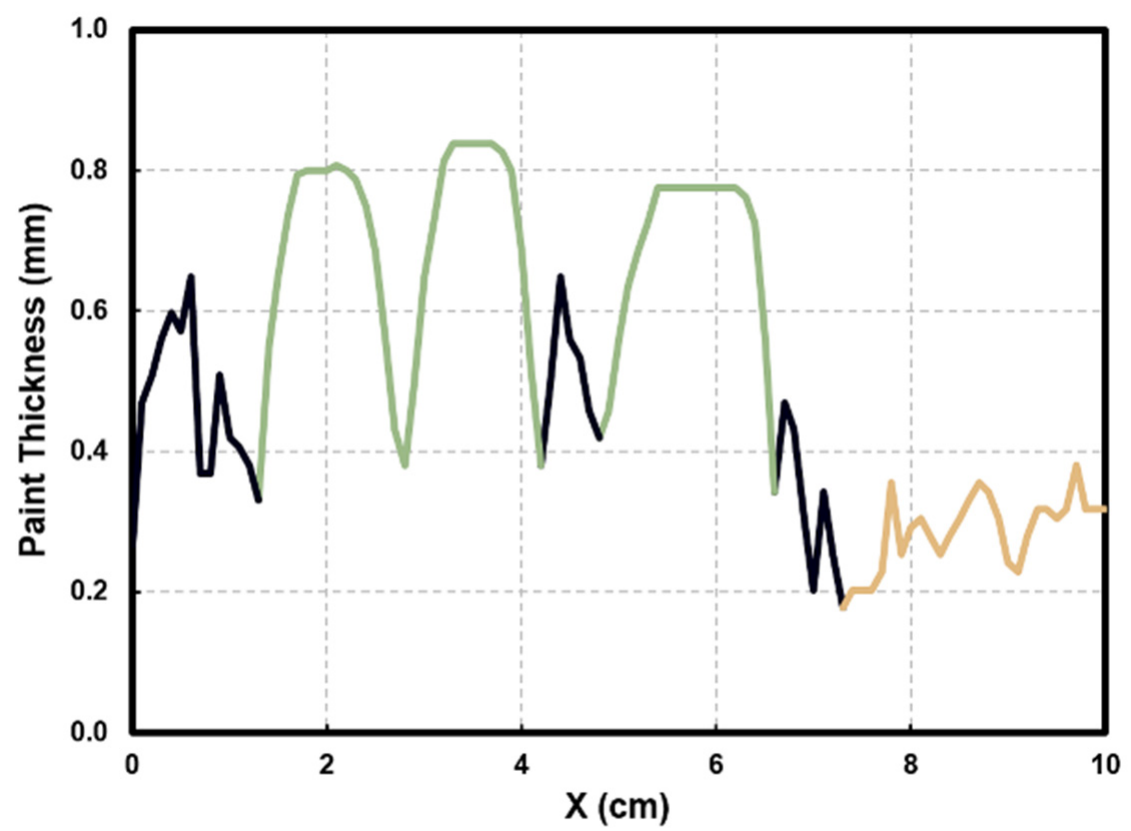

Figure 5. The pigment thickness along a line in the painting corresponding to $Z$ grid line 9 . The thickness plot is color coded to the paint's RGB pigment color.

For spectral identification, it is preferable that the sweep width be wide enough for the signal to start and end at zero signal. However, because these are LFEPR spectra where the absorption widths can be comparable to the sweep width and the signal greater than zero at the start of the scan, a method of reproducibly overlaying spectra was necessary. The decided-upon method was one where all spectra were offset to start at zero signal at zero magnetic field and normalized such that the largest negative signal values in the spectra were equal. The offset, then normalized, EPR spectra for the four pigments are presented in Figure 5. The four spectra present the similarities and differences in the spectra, and the challenges of identifying them. These spectra are the $R_{i}$ spectra introduced in the background section. For the matrix algorithm, the four magnetic field values for $m=1,2,3$, and 4 in Equation (6) were 8.69, 12.79, 17.39, and $28.64 \mathrm{mT}$ in these spectra. The vertical dashed lines in Figure 6 indicate the location of these field values. These field values were chosen to give unique, non-zero points in the spectra. They are sufficient to perform the identification, but may not be optimal. 


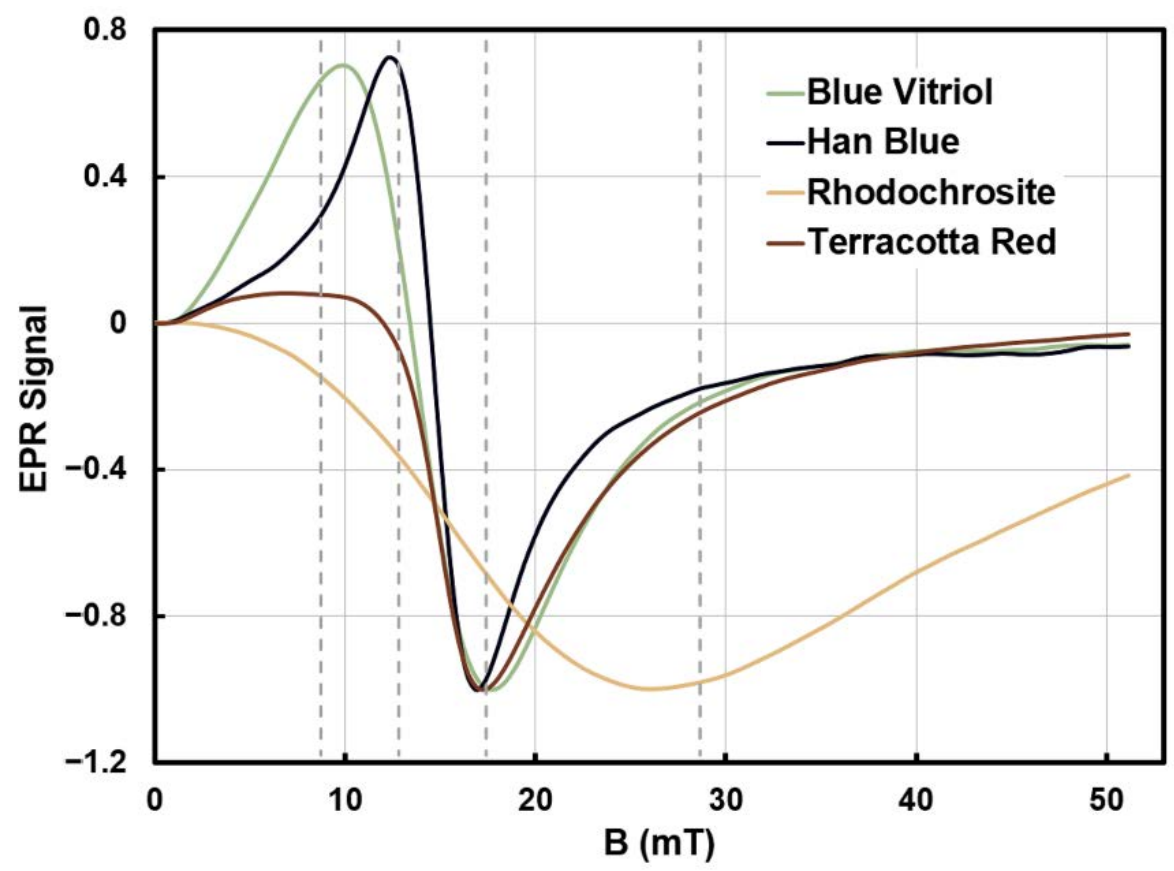

Figure 6. LFEPR reference spectra of the four pigments in the painting. Spectra are offset to start at zero signal and normalized such that the largest negative signal values were equal. Dashed vertical lines indicate the field values used in the matrix algorithm.

Figure 7 presents the segmented images by the three pigment identification algorithms. Also presented in this figure are discrepancy images where there is a difference between the truth image (Figure 4) and an algorithm image. Image pixelation is a consequence of the $29 \times 30$-point sampling grid. The algorithms prevented a pixel from belonging to more than one pigment class. All three algorithms presented a recognizable image that is comparable to the original painting and the truth image. Differences between the outputs of the algorithms in the pixels identified as a certain pigment were less than five pixels in all cases. Incorrectly identified pixels were primarily associated with boundary locations where two or more pigments occupied a sampling region and a consequence of the algorithm picking the larger signal over the larger visible surface area pigment. This can be seen at the BV-HB and HB-RC boundaries. One exception can be found at grid location $(z, x)=(21,19)$ by the least squares algorithm. The truth image presents this location as BV, but it was identified as TR. Since there is no TR in the painting at this point, the discrepancy is attributed to the simplicity of this algorithm and the portions of RC and BV present being interpreted as TR by the algorithm. The matrix and interior-point algorithms identified this location correctly as BV.

The metrics for the four pigments by the three algorithms are summarized in Table 2. Of the 870 grid locations on the painting, 210,343, 270, and 47 are, respectively, assigned to $\mathrm{BV}, \mathrm{HB}, \mathrm{RC}$, and TR in the truth image. All algorithms had a true positive rate of 0.95 or better and a true negative rate of 0.98 or better for all pigments. These numbers are an indication of the high sensitivity and specificity of all three algorithms. These two metrics varied little between the algorithms for a given pigment. Similarly, there was very little discrepancy between the algorithms in the number of false positives and false negatives for a given pigment. In general, rhodochrosite was the most correctly identified pigment by all the techniques. This was followed by terracotta red and blue vitriol. Han blue was incorrectly identified the most with a false positive rate of 0.01 and a false negative rate of 0.04 to 0.05 by the algorithms. 

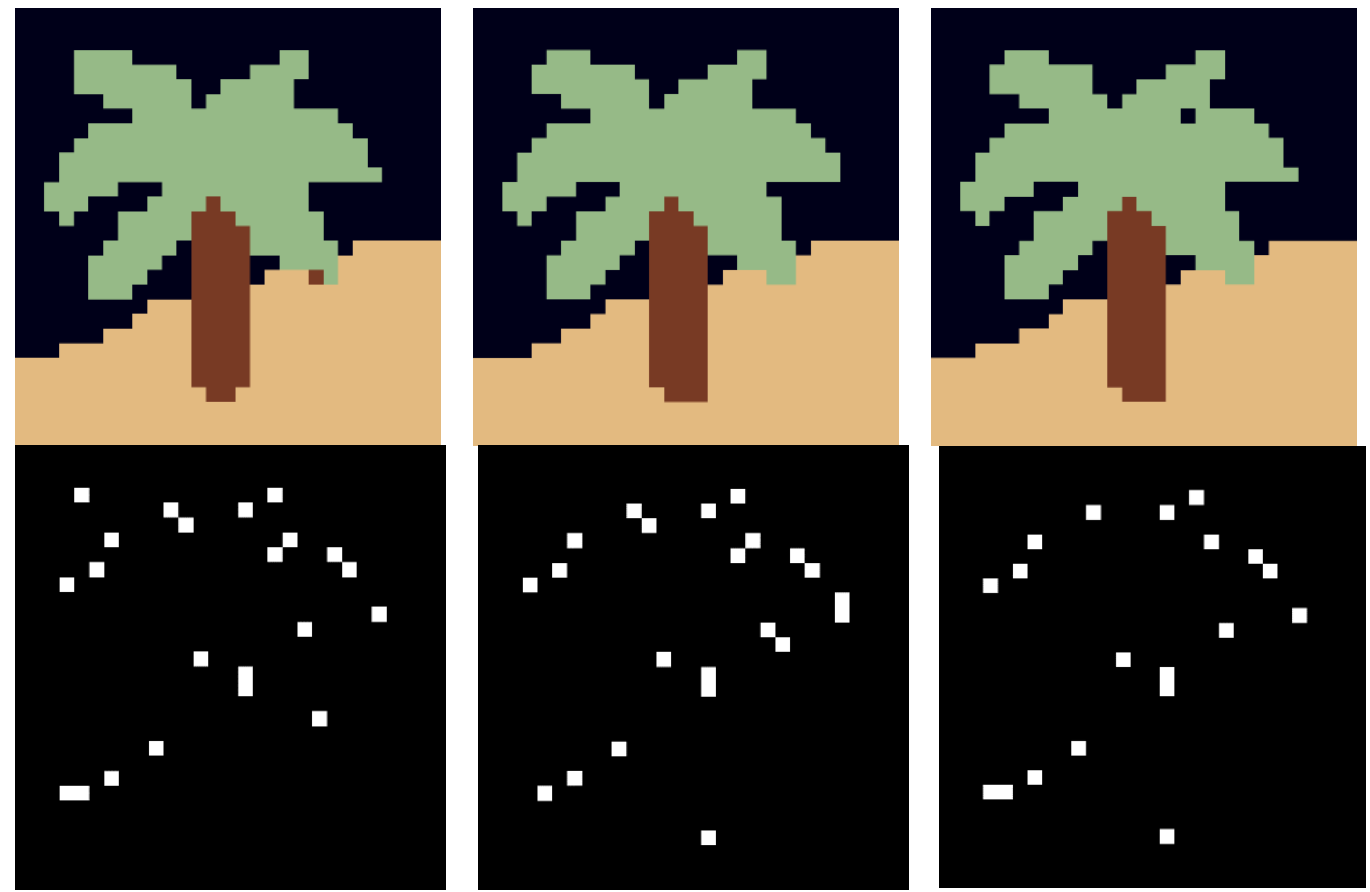

Figure 7. Colorized composite (row one) and grayscale discrepancy (row two) images from the least squares (left), matrix (center), and interior-point (right) algorithms.

Table 2. Evaluation metrics for the identification algorithms.

\begin{tabular}{cccccc}
\hline \multirow{2}{*}{ Algorithm } & Metric & \multicolumn{4}{c}{ Pigment } \\
\cline { 3 - 6 } & & Blue Vitriol & Han Blue & Rhodochrosite & Terracotta Red \\
\hline \multirow{5}{*}{ Matrix } & TP/P & 0.98 & 0.95 & 1.00 & 1.00 \\
& FP/N & 0.02 & 0.01 & 0.01 & 0.00 \\
& FN/P & 0.02 & 0.05 & 0.00 & 0.00 \\
& TN/N & 0.98 & 0.99 & 0.99 & 1.00 \\
\hline \multirow{5}{*}{ Least Squares } & TP/P & 0.97 & 0.95 & 1.00 & 1.00 \\
& FP/N & 0.02 & 0.01 & 0.01 & 0.00 \\
& FN/P & 0.03 & 0.05 & 0.00 & 0.00 \\
& TN/N & 0.98 & 0.99 & 0.99 & 1.00 \\
\hline \multirow{5}{*}{ Interior-Point } & TP/P & 0.98 & 0.96 & 1.00 & 1.00 \\
& FP/N & 0.01 & 0.01 & 0.01 & 0.00 \\
& FN/P & 0.02 & 0.04 & 0.00 & 0.00 \\
& TN/N & 0.99 & 0.99 & 0.99 & 1.00 \\
\hline
\end{tabular}

Similarities between the less than perfect metrics from an algorithm for a given pigment are attributed to EPR spectral imperfections from noise. Since most discrepancies are seen at the boundaries between pigments, where two pigments occupy the elliptical sampling region, we attribute them to variations in the topography of the surface of the painting and the use of a flat RF sample coil mounted in a flat housing approximately equal to the size of the painting. Since the limit of detection increases as distance from the sample coil increases, i.e., the coil creates less signal the further away the sample is from its surface, the pigment closest to the sample coil will have the dominant signal of the two. We estimated that the signal from a pigment covering as little as $40 \%$ of the ellipse area and in contact with the coil would dominate over a pigment offset from the surface and occupying $60 \%$ of the ellipse. The results indicate that this may be too conservative an estimate.

For this four-pigment painting, it is possible with the matrix method to scan the painting at just four magnetic field values rather than 100 . The protocol would be to raster 
the mouse over the grid locations at four B value instead of incrementing the MOUSE location and scanning the entire sweep width. This would significantly decrease the data acquisition time.

\section{Conclusions}

The results of this study present art conservators and historians with another analytical technique for identifying paramagnetic pigments and mapping their spatial distribution in a painting. The EPR MOUSE can noninvasively collect LFEPR spectra from grid points on a painting either by moving the painting over the MOUSE or the MOUSE over the painting. These spectra can identify the pigment at each grid location on the painting. A dense grid of identified pigments can be presented as a spatial map or color image of the pigments in the painting.

All three spectral identification algorithms produced in an unsupervised manner images that closely resembled the original painting and very closely matched the truth image. The metrics used to compare the pigment maps from the three algorithms to the truth image were exceptionally good. For all pigments by all algorithms, the true positive rate was at least 0.95 , and the true negative rate 0.98 . Similarly, the false positive and false negative rates were less than or equal to 0.02 and 0.05 , respectively.

We believe the interior-point was the most robust algorithm followed by the least squares and matrix algorithms. The matrix method was least robust because it depended on only four spectral points and was the most sensitive to spectral noise. The least squares algorithm was the simplest and worked surprisingly well. All three algorithms are scalable to a larger number of pigments; however, performance will depend on the uniqueness and quality of the spectra. Quantitative results should be possible with flatter (less impasto) painting surfaces or the redesign of the MOUSE surface.

This study is part of a methodical approach to developing LFEPR spectroscopy for noninvasively studying paintings. Earlier studies focused on identifying single pigments [21], mixtures of two pigments [22], pigment layers [22], imaging of one component [23], and now mapping the spatial distribution of four pigments. The presented analytical technique is promising for application in topics related to the preservation of cultural her-itage, since it is non-destructive and the designed system can operate automatically. In the future, we intend to examine underpaintings, expand mapping to more pigments, and apply our techniques to paintings with cultural heritage significance.

Author Contributions: Conceptualization, J.P.H. and H.W.; methodology and software, S.M. and H.W.; validation, S.M. and H.W.; formal analysis, S.M., H.W. and J.P.H.; painting, H.W.; writingoriginal draft preparation, J.P.H.; writing-review and editing, S.M., H.W. and J.P.H.; supervision, J.P.H. All authors have read and agreed to the published version of the manuscript.

Funding: This research received no external funding.

Acknowledgments: The authors thank Olivia Kuzio for helpful comments during the preparation of the paper.

Conflicts of Interest: The authors declare no conflict of interest.

\section{References}

1. Rowe, M.W. Dating of rock paintings in the Americas: A word of caution. In Proceedings of the International Federation of Rock Art Organizations Congress, Tarascon-sur-Ariège, France, 6-11 September 2010; pp. 573-584.

2. Moretto, L.M.; Orsega, E.F.; Mazzocchin, G.A. Spectroscopic methods for the analysis of celadonite and glauconite in Roman green wall paintings. J. Cult. Herit. 2011, 12, 384-391. [CrossRef]

3. Bensimon, Y.; Deroide, B.; Clavel, S.; Zanchetta, J.-V. Electron spin resonance and dilatometric studies of ancient ceramics applied to the determination of firing temperature. Jpn. J. Appl. Phys. 1998, 37, 4367-4372. [CrossRef]

4. Presciutti, F.; Capitani, D.; Sgamellotti, A.; Brunetti, B.G.; Costantino, F.; Viel, S.; Segre, A. Electron paramagnetic resonance, scanning electron microscopy with energy dispersion X-ray spectrometry, X-ray powder diffraction, and NMR characterization of iron-rich fired clays. J. Phys. Chem. B 2005, 109, 22147-22158. [CrossRef] 
5. Warashina, T.; Higashimura, T.; Maeda, Y. Determination of the firing temperature of ancient pottery by means of ESR spectrometry. . Brit. Mus. Occas. Pap. 1981, 19, 117-128.

6. Mangueira, G.M.; Toledo, R.; Teixeira, S.; Franco, R.W.A. A study of the firing temperature of archeological pottery by X-ray diffraction and electron paramagnetic resonance. J. Phys. Chem. Solids 2011, 72, 90-96. [CrossRef]

7. Christiansen, M.B.; Sørensen, M.A.; Sanyova, J.; Bendix, J.; Simonsen, K.P. Characterisation of the rare cadmium chromate pigment in a 19th century tube colour by Raman, FTIR, X-ray and EPR. Spectrochim. Acta Part A Mol. Biomol. Spectrosc. 2017, 175, 208-214. [CrossRef]

8. Gobeltz, N.; Demortier, A.; Lelieur, J.P.; Duhayon, C. Correlation between EPR, Raman and colorimetric characteristics of the blue ultramarine pigments. J. Chem. Soc. Faraday Trans. 1998, 94, 677-681. [CrossRef]

9. Reddy, S.L.; Reddy, G.U.; Reddy, T.R.; Thomas, A.R.; Reddy, R.R.S.; Frost, R.L.; Endo, T. XRD, TEM, EPR, IR and Nonlinear Optical Studies of Yellow Ochre. J. Laser Opt. Photonics 2015, 2, 120. [CrossRef]

10. Orsega, E.F.; Agnoli, F.; Mazzocchin, G.A. An EPR study on ancient and newly synthesised Egyptian blue. Talanta 2006, 68, 831-835. [CrossRef]

11. Raulin, K.; Gobeltz, N.; Vezin, H.; Touati, N.; Lede, B.; Moissette, A. Identification of the EPR signal of S2 ${ }^{-}$in green ultramarine pigments. Phys. Chem. Chem. Phys. 2011, 13, 9253-9259. [CrossRef]

12. Polikreti, K.; Maniatis, Y.; Bassiakos, Y.; Kourou, N.; Karageorghis, V. Provenance of archaeological limestone with EPR spectroscopy: The case of the Cypriote-type statuettes. J. Archaeol. Sci. 2004, 31, 1015-1028. [CrossRef]

13. Polikreti, K.; Maniatis, Y. Distribution of changes of Mn(II) and Fe(III) on weathered marble surfaces measured by EPR spectroscopy. Atmos. Environ. 2004, 38, 3617-3624. [CrossRef]

14. Polikreti, K.; Maniatis, Y. A new method for the provenance of marble based on EPR spectroscopy. Archaeometry 2002, 44, 1-21. [CrossRef]

15. Attanasio, D.; Capitani, D.; Federici, C.; Segre, A.L. Electron spin resonance study of paper samples dating from the fifteenth to the eighteenth century. Archaeometry 1995, 37, 377-384. [CrossRef]

16. Zoleo, A.; Nodari, L.; Rampazzo, M.; Piccinelli, F.; Russo, U.; Federici, C.; Brustolon, M. Characterization of pigment and binder in badly conserved illuminations of a 15th-century manuscript. Archaeometry 2014, 56, 496-512. [CrossRef]

17. Dietemann, P.; Kälin, M.; Zumbühl, S.; Knochenmuss, R.; Wülfert, S.; Zenobi, R. A Mass Spectrometry and Electron Paramagnetic Resonance Study of Photochemical and Thermal Aging of Triterpenoid Varnishes. Anal. Chem. 2001, 73, 2087-2096. [CrossRef]

18. Ottaviani, M.F.; Geniali, A.; Cangiotti, M.; Mangani, F. EPR analysis of the interacting properties and the degradation over time and irradiation of mastic resin used for painting protection. Res. Chem. Intermed. 2002, 28, 117-130. [CrossRef]

19. Switala, L.E.; Ryan, W.J.; Hoffman, M.; Santana, S.J.; Black, B.E.; Hornak, J.P. A Wide-Line Low Frequency Electron Paramagnetic Resonance Spectrometer. Concepts Magn. Reson. Part B Magn. Reson. Eng. 2017, 47B, e21355. [CrossRef]

20. Switala, L.E.; Black, B.E.; Mercovich, C.A.; Seshadri, A.; Hornak, J.P. An Electron Paramagnetic Resonance Mobile Universal Surface Explorer. J. Magn. Reson. 2017, 285, 18-25. [CrossRef]

21. Javier, S.; Hornak, J.P. A Non-Destructive Method of Identifying Pigments on Canvas Using Electron Paramagnetic Resonance Spectroscopy. J. Am. Inst. Conserv. 2018, 57, 73-82. [CrossRef]

22. Bogart, E.A.; Wiskoski, H.; Chanthavongsay, M.; Gupta, A.; Hornak, J.P. The noninvasive analysis of paint mixtures on canvas using an EPR MOUSE. Heritage 2020, 3, 140-151. [CrossRef]

23. Switala, L.E.; Ryan, W.J.; Hoffman, M.; Brown, W.; Hornak, J.P. Low Frequency EPR and EMR Point Spectroscopy and Imaging of a Surface. Magn. Reson. Imaging 2016, 34, 469-472. [CrossRef]

24. Fletcher, L.M.; Barsotti, J.B.; Hornak, J.P. A multispectral analysis of brain tissues. Magn. Reson. Med. 1993, 29, 623-630. [CrossRef]

25. Kleynhans, T.; Patterson, C.M.S.; Dooley, K.A.; Messinger, D.W.; Delaney, J.K. An alternative approach to mapping pigments in paintings with hyperspectral reflectance image cubes using artificial intelligence. Herit. Sci. 2020, 8, 1-17. [CrossRef]

26. Poole, C.P., Jr. Electron. Spin Resonance, 2nd ed.; John Wiley \& Sons: New York, NY, USA, 1983; p. 780.

27. Potra, F.A.; Wright, S.J. Interior-point methods. J. Comput. Appl. Math. 2000, 124, 281-302. [CrossRef]

28. Hornak, J.P. The development of low frequency EPR spectroscopy for studying objects with cultural heritage significance. In Spectroscopic Techniques for Archaeological and Cultural Heritage Research; Shukla, A.K., Ed.; IOP Publishing: Bristol, UK, 2020; Chapter 3; pp. 1-54.

29. Fawcett, T. An introduction to ROC Analysis. Pattern Recognit. Lett. 2006, 27, 861-874. [CrossRef] 